\title{
Interactive comment on "Effects of Seasonal Snow Cover on Hydrothermal Conditions of the Active Layer in the Northeastern Qinghai-Tibet Plateau" by Ji Chen et al.
}

\author{
A. Rist (Referee) \\ armin.rist@giub.unibe.ch \\ Received and published: 4 August 2016
}

General comments:

This discussion paper addresses the interesting and for geocryology important topic of the snow cover's effect on the hydrothermal regime of the active layer. While this topic was already intensively studied in Arctic and Alpine permafrost regions, little attention was paid to it in a high-altitude arid steppe underlain by permafrost such as the QinghaiTibet Plateau.

The literature review given in the introduction is quite comprehensive for a paper. It could be sharpened by reducing the number of mentioned studies to those which can 
be clearly and closely related to the present study.

The objectives or research questions of the study should be expressed more clearly. The applied methods are suitable to achieve the objectives (as indirectly derived from the introduction). One problem is that not all parameters were measured for the same period of time which makes comparisons and correlations difficult. It is in the nature of field research that improvement opportunities for methods only shape out during the measurements and that the analysis of the gained data can require to measure additional parameters or to apply further methods. However, to make the paper better understandable I suggest to give an overview in a figure or table when which parameter was measured.

The results are overall structured and presented logically. Some information given in the results would fit better under the methods (see specific comments). Single argumentations in the results and discussion are partly not easy to follow for the reader and could thus be presented more consecutive and structured. The discussion is structured by questions used as headings. This is an elegant way to show concisely what the reader can expect from this chapter. One subchapter is dedicated to the thermal and hydrological effects of the snow cover on the active layer, respectively. However, to discuss the interrelations between the thermal and hydrological effects would be interesting and complete the discussion. The extension of the discussion by these interrelations could be compensated by restricting the contents to those which are clearly and closely related to the topic of this paper.

The conclusions comprise the study's findings concerning the snow regime, its effects on the thermal and hydrological regime of the active layer, respectively. Also here a conclusion relating the heat and water balance being influenced by the snow cover would be desirable.

Concerning the English language I can neither assess nor give advice for improvements as I am not a native speaker as well. However, I recommend to ask a native

Printer-friendly version

Discussion paper
Interactive

comment 
speaker to smoothen the English.

Although I checked minor revisions my comments are quite numerous. However, they are not severe concerning the content. After the revision I strongly recommend to accept this paper for publication in the journal TC.

Specific comments:

Line 13: Make transition smoother from first to second sentence;

Line 17: An event cannot be thick $\rightarrow$ find correct formulation;

Line 19: Not clear; could it be like this: cooling of the active layer will be increased if the snow is removed all year round and even more if it is removed in winter, spring and autumn, but left in summer;

Line 21: Don't understand; was the snow removed only some weeks in autumn and then left? Above you wrote that the snow was removed the whole year or at least whole seasons;

Lines $22,87, \ldots$ : I suggest either soil moisture or soil water content, but not soil moisture content;

Line 24: You already mentioned the decrease of the soil water content in the last sentence of (2);

Line 25: Less compared to what?

Lines 26-28: First mention that you observed lower soil temperatures and higher soil water contents at the NSS than at the SRS; then come up with your interpretation; another reason is the higher albedo of snow; look for literature on this topic;

Lines 36-37: Do all these authors in brackets give statements to all topics you mentioned in the sentence before? If not, please put each citation directly after the corresponding topic to make clear which content originates from which author/s; 
Line 39: 'Alpine' is an adjective, but can not be used in addition as a noun as 'Arctic' or 'Antarctic'; furthermore, 'Alpine' is usually related to the European Alps, while 'alpine' identifies an altitudinal belt in mountains;

Line 39: Do all these authors in brackets give statements to all topics you mentioned in the sentence before? If not, please put each citation directly after the corresponding topic to make clear which content originates from which author/s;

Line 47: Only for fresh snow; for old snow the albedo can drop below 80\%;

Lines 49-50: 'specific latent heat of fusion at the transition from ice to liquid water' rather than 'latent heat' as the released energy is related to a given mass; where did you get the value $335 \mathrm{~kJ} / \mathrm{kg}$ from?; in all physics books I know it is $334 \mathrm{~kJ} / \mathrm{kg}$;

Line 50: Delays compared to what? To an imaginary lower value of the specific latent heat of fusion?

Lines 53, 54: Only 'snow' instead of 'snow cover' because a cover has a thickness but here you refer to a material constant;

Line 55: Do you mean the European Alps? there are also other Alps like those e.g. in New Zealand;

Line 55: I suggest '.. that the daily rate of the increase in the thermal conductivity coefficient of snow is ...; I guess this increase takes place during the melting period, but you have to tell the reader otherwise it is not clear.

Line 67: Name the country where these mountains are;

Line 69: Regarding 'temperature difference': as average over the whole winter season?

Line 72: Why 'Pole'? Do you mean ....'in the arctic and subarctic regions'?

Printer-friendly version

Line 77: Well, there is also an insulation effect below the given values, which rather refer to a (nearly) complete thermal insulation of the ground from the atmosphere by 
the snow cover;

Line 85: More than what?

Line 89: 'the permafrost shell thickness of the surface layer' $\rightarrow>$ I don't understand what you mean by this; please highlight;

Lines 91-94: What is the main finding of this study related to your study?

Lines 95-96: You already explicated this statement in lines 43-59; avoid repetitions; you could start the sentence like: While previous studies investigated ..., in this study ...;

Line 98: Not 'mountain island permafrost' but 'patchy mountain permafrost';

Line 100: Please explain these findings more detailed; Lines 101-109: Clearly tell the objectives or research questions of your study somewhere after the literature review and the gaps of knowledge;

Line 105: Do you mean '... down to a depth of ...?';

Line 110: I suggest simply: '2. Methods';

Line 111: 'Description of' is dispensable, omit it;

Line 121: Label the figure parts using small letters $(a, b, c)$, refer to them in the figure caption (below the figure) an give explanations only there;

Line 122 (Fig. 1a): Red points for cities are quite dominant compared to the landscape and especially the monitoring site; I recommend to make at least the monitoring site more visible (e.g. red frame);

Line 122 (Fig. 1a): The unit is usually given in [...]; would also do it like this here and throughout the manuscript (please check in all figures, tables and in the text where you give the unit after a numerical property);

Line 122 (Fig. 1a): I recommend to use the color gradient for the elevation inversely: 
green for lowest and brown for highest altitudes (more common and intuitive);

Line 122 (Fig. 1a): Add a scale bar;

Line 124 (Fig. 1b): Could you show the topographic map or an aerial photograph in the background to get a better impression of the soil surface coverage?

Line 124 (Fig. 1b, Legend): 'Monitoring site' is exactly the same expression as for the whole monitoring site you show in Fig. 1a - this is misleading; I suggest to use the word 'monitoring plot' for the areas you called NSS and SRS which then change to NSP and SRP;

Line 127: Give meaning of all abbreviations; figures and tables (in combination with their caption) have to be understandable by its own; check for abbreviations in figures and tables throughout the manuscript;

Lines 130-131: I suggest '..., the air temperature in the region ranged between minimum values of $-32.6^{\circ} \mathrm{C}$ and maximum values of $22.9^{\circ} \mathrm{C}$ while the average annual value was ...;

Line 131: Why do you give a range for the average annual values but not for the minimum and maximum annual values?

Line 134: I suggest: 'mean annual gust speed (measured half-hourly)';

Line 134: What is the name of the river? Also show it in Fig. $1 \mathrm{a}, \mathrm{b}$;

Lines 148 and 150 (Fig. 2a and 2b): 'Total depth' means what? Depth of the borehole, $6 \mathrm{~m}$ ?

Lines 148 and 150 (Fig. 2a and 2b): Regarding 'Column 1:100' $\rightarrow$ On my screen (at a view size of $100 \%) 1 \mathrm{~m}$ is equivalent to $6 \mathrm{~mm}$, so the scale $1: 100$ might be correct in the original figure but it was changed when implanting in this document;

Lines 148 (Fig. 2a): Regarding second layer from top $\rightarrow$ I can hardly believe that the 
soil can be saturated at a water content of $2.6 \%$ (at $2.1 \mathrm{~m}$ depth) within this layer which you generally call water saturated (equivalent for third layer from top in Fig. 2b);

Line $153 \ldots$. . You are describing the equipment here but the heading of 2.1 only refers to the monitoring site;

Line 157: Here you just refer to NSS and SRS. Explain your concept of investigating the effect of the snow cover by comparing one site with a natural snow cover with another one where you artificially removed the snow; this is essential for the understanding of the whole study; and describe it before you come to the measurement equipment;

Line 160: Ground temperature at which depth?

Line 160-161: Do you mean: maximum duration of the snow season?

Line 162: In line 153 you already mentioned a ground temperature equipment; how is it related to the probe you mention here?

Line 167: Why did you measure the soil thermal flux only at the NSS, but not at the SRS?

Line 170 (Table 1): Here you list the measurement parameters wind speed, rel. air humidity, air pressure, precipitation, albedo in the methods but you don't present them in the results or discussion; If you don't need them for your paper delete them in Tab. 1 ;

Line 170 (Table 1): As not all parameters were monitored for the same period of time it would be helpful to give the monitoring period for each parameter, either in this table or even more vivid in a figure;

Line 170 (Table 1): To me the following order of columns from left to right would be understandable more intuitively: Type of probe, Number of probes (NSS [as reference first], SRS), Measuring range, Measuring accuracy, Brand, Model;

Line 170 (Table 1): Please tell the measuring interval(s) of the different parameters; 
Line 170 (Table 1): Why did you use different soil moisture and albedo probes at SRS and NSS? This makes it more difficult to compare the measured values;

Line 170 (Table 1): for the soil moisture probes you have to say if \% refers to the gravimetric water content (as in Fig. 2) or the volumetric one;

Line 175: Not clear. Do you mean you used the measured air temperature to correct the signal of the ultrasonic sensor measuring snow depth?

Line 180: All means all, so you don't need to list some probes;

Line 182: Specify the logger by the brand, at least;

Line 186: But I can see a fence around the SRS in Fig. 3b and c; so what do you mean by snow fence?

Line 188: How could you guarantee that the SRS wasn't covered by snow again due to wind after snow removal?

Line $193 \ldots$. . A lot of information on the acquisition of soil temperature data was already given under 2.1 ; the information given here would fit to table 1 ; temperature measurement is a standard technique; why do you describe it here so extensively?

Line 205: See comments on the heading of 2.2 (Line 193) and transfer to 2.3;

Line 212-213: Did you dig the test pit and push the rods of the probes horizontally into a side wall or did you burry the probes in layers?

Line 222: Repetition, information already in Tab. 1;

Line 223: What do you mean by hydrothermal probes? The soil temperature and moisture probes together? Did you have to dig into the active layer to install the mast onto which the ultrasonic snow depth sensors is mounted or why do you mention digging in connection with the snow depth measurements?

Line 228: Do you mean because of the calibration for unfrozen conditions by the manu- 
facturer? How did you get the true value? By gravimetric water content measurements using soil probes? Or do you stress the fact that the probes measure the liquid water content but not the frozen water content? Not clear;

Line 237: Do you mean accumulated in total?

Line 245: I question if the snow depth can be determined with a resolution of $1 \mathrm{~mm}$ (surface hoar on the snow can be thicker than $1 \mathrm{~mm}$ );

Lines 251-252: '... snow cover remained 5 days at maximum in more than $90 \%$ of the snowfall events, ...';

Line 253: In the same sentence you say that the snow cover would have been lasting not more than 5 days in general - this is a contradiction; instead of the words 'generally' and 'typically' try to quantify in the statements;

Line 270 (Tab. 2): Data of air temperature and radiation averaged over the seasons would be helpful here as you argue in the text with these parameters;

Line 272: As you define the depth negative in Fig. 7 the maximum depth would be 0; so either you can say something like 'the most negative depth' or you use a positive sign for depth values in Fig. 7;

Line 277: I question if the ALT can be determined with a resolution of $1 \mathrm{~mm}$;

Lines 284-288: This sentence needs to be related to the ground temperature measurements of this study;

Line 286: Regarding 'at any depth' $\rightarrow>$ the mean annual ground (rather than soil) temperature MAGT refers to the depth of the zero annual amplitude (see Wu and Zhang, 2008, caption of Table 2);

Line 291: Do you mean the extremes of daily air temperatures within a year? This must become more clear because the extremes of the air temperature within a day must not be excluded to estimate the daily geothermal propagation depth; 
293: Did you determine the daily geothermal propagation depth? If not, please cite the publication where you got this information from;

Line 294: It depends on the purpose; what do you want to say with these data?

Lines 293-296: I understand the sentence up to '... single time', but the words thereafter (several times, even partial time of each day) I can't logically relate to the rest of the sentence. Maybe 'or' is missing before 'even'?

Line 303 (Fig. 8): The colors in Fig. 8 should be harmonized with those in Fig. 10 regarding their meaning;

Line 303 (Fig. 8f): How do you interprete that the MAGT-curves are bent towards lower temperatures above 1.5 m? Compare to Fig. 3 in Smith \& Riseborough (2002): Climate and the limits of permafrost;

Line 303 (Fig. 8f): I recommend not to choose blue and red lines in Fig. 8d as these colors are used in a different meaning in Fig. $8 \mathrm{a}-8 \mathrm{e}$;

Lines 304-307: In line 304 you say 'annual' for the entire Fig. 8, but later you say that the temperature profiles are averaged over seasons!

Lines 309-311: This should additionally be described in methods;

Line 323: 'In terms of yearly temperature,' is dispensable;

Lines 323-325: As first sentence of this paragraph I suggest: 'The mean annual soil temperature at $0.5 \mathrm{~m}$ depth was $0.8^{\circ} \mathrm{C}$ higher for the NSS than the SRS while the temperature difference between the two sites decreased with depth being approximately zero below 2 m depth.';

Lines 325-326: According to Figure 8e they are approximately the same compared to the layer above $1.6 \mathrm{~m}$ !

Line 327: What do you mean by 'generally' here? Average difference over the whole 
depth profile or only below $1.6 \mathrm{~m}$ ? Not clear;

Lines 328-329: This sentence in the present form belongs to methods but not to results; don't repeat methods in results;

Lines 323-333: Not clear! I suggest: 'The MAAT in Yashatu was $-4.5^{\circ} \mathrm{C},-3.4^{\circ} \mathrm{C}$, and $-3.9^{\circ} \mathrm{C}$ in 2012, 2013 and 2014, respectively, indicating ...;

Line 333: Where did you get the value for 2012 from? According to Fig. 4 you only measured the air temperature since December 2012; you have to cite the source if it is not your own measurement;

Lines 338-339: This sentence is only valid for NSS, tell the reader;

Lines 340-341: Regarding '..., there is ...' $\rightarrow$ Where? Do you mean at the SRS? So you have to tell it the reader. However, I see something different in Fig. 9: besides the small L-shaped area at SRS with a water content above $40 \%$ in 2013 the water contents at NSS are higher throughout the depth profile and throughout the monitoring period;

Line 342: 'based on CS616' does not belong to results but to methods and shouldn't be repeated;

Line 342: It can, but does it or does it not?

Lines 343-345: There appeared strong changes during redistribution, but if you direclty compare the water content profile at NSS in Oct. 2013 and Oct. 2014 I roughly agree although I can see an increase from 30 to $40 \%$ between about $1.1 \mathrm{~m}$ and $1.7 \mathrm{~m}$ depth $\rightarrow$ has to be described more clearly;

Line 345: Is the class with the highest water content from 40 to $70 \%$ ? Please indicate in the color bar in Fig. 9. However, a vol. water content of $70 \%$ in gravel (as stated in Fig. 2) is very unlikely to me even for saturation (maybe in clay it would be possible);

Line 349: Just to describe what you can see anyway in Fig. 10 doesn't give an addi- 
tional benefit; add information in the text you can't directly see in a Fig. itself;

Lines 354-355: Sentence is too complicated to express the facts;

Line 356: Give a depth range as in Fig. 10;

Line 358: 'first' and 'then' means from 2012-2013 and from 2013-2014, respectively; express it more clearly;

Line 359 (Fig. 9): What is the upper limit of the highest class above $40 \%$ ? Please, indicate on the color bar;

Line 359 (Fig. 9): How do you explain the pronounced steps in the water content profiles, especially in 2013 ?

Line 360 (Fig. 9, caption): ... 'based on CS616' is an information regarding the methods and must not be repeated here;

Line 360 (Fig. 9, caption): In Table 1 you say that soil moisture at NSS was measured using a probe called SM300 but not CS616;

Lines 364-369: Too complicate; just say that you linearly interpolate between the point measurements using the soil moisture probes;

Lines 364-379: This information would be more adequate in the methods;

Line 379: A range is from a minimum to a maximum value; here you mean the difference;

Line 382: Not 'in 2013 and 2014' but 'from 2012 to 2013 and from 2013 to 2014';

Line 385: Mark figure parts either with $a$ and $b$ and refer to them in the caption or with NSS and SRS but not both;

Line 394: See comment on line 47 and 51;

Line 397: Cite these studies; 
Line 399: It did in case of ASR-1, but not for ASR-2;

Line 402: To see the effect of the snow cover at the SRS by comparing the 3 years 2012, 2013 and 2014 (i.e. BSR, ASR-1, ASR-2) is hardly possible as you have 3 samples (years) only, but 2 factors (air temperature and snow cover);

Lines 400-403: You cannot directly compare ASR-2 (i.e. SRS 2 years after snow removal) with NSS because the observation period of ASR-2 was 2013.12-2014.11 (line 311) while it was 2014.3.1-2015.2.28 (line 300) for NSS; however, you can compare SRS and NSS in the same period of monitoring as shown in Fig. 8e. Maybe you meant this, but it is not clear;

Line 407: Are these results yours? If not, cite the publication in the same sentence;

Line 409: This finding means that the ground cooling effect due to the change of the albedo by the first snow cover in autumn is less effective than the cooling due to the stronger heat dissipation without snow; finally, the thermal insulation (and during this season warming) effect of the snow cover overbalanced the cooling effect due to the higher albedo of snow in this study;

Lines 414-415: '... when the ground temperature is higher than the air temperature'?

Line 418: Table 3 shows results and should thus be presented in chapter 3 , but not only in the discussion;

Line 418 (Table 3, caption): This is again another period of time than for the collection of the other data (soil temperature, air temperature, water content); so it is difficult to relate them to each other;

Line 424: Which areas?

Printer-friendly version

Line 426: not correct: latent heat is released by freezing and by condensation, but not by a decrease in the water content; if the soil water content in autumn is less than the year before, also the latent heat released during freezing will be less; is it this point you 
wanted to make? Then make it clearer, please;

Line 427: See comment on line 50;

Line 429: But at $4000 \mathrm{~m}$ a.s.l. altitude the pressure is much lower $\rightarrow$ adapt the following calculations to a realistic atmospheric pressure at $4000 \mathrm{~m}$ a.s.l.;

Interactive

Line 431: How did you determine this heat capacity? Please show that it is realistic using the content of mineral material, ice and water;

Line 430-433: Has to be explained more clearly. Just by reducing the water content the temperature won't decrease! However, the heat to be extracted from $1 \mathrm{~m} 3$ of soil to freeze the water equivalent to a $\mathrm{VWC}=1 \%$ is $3350 \mathrm{~kJ}$. If the same heat would be extracted from the same body of soil without freezing (i.e. if already al the water is frozen or al the water remains liquid) a temperature decrease of $1.5^{\circ} \mathrm{C}$ would occur. For the heat of vaporization the argumentation is equivalent;

Line 435 ...: I can't see that you used the thermal flux data to verify 'this phenomenon' explained above. I would just argue that at higher water contents more heat has to be extracted for freezing than in drier soils. The atmosphere can take up only a given amount of heat under given meteorological conditions. After freezing, in driers soils more heat that can be dissipated is left for ground cooling resulting in lower temperatures;

Line 438: To make these four stages more visible I suggest a diagram rather than a table;

Line 444: It should be like this, but Table 3 shows the opposite! You argued for all other months. I would say the higher/lower values in February/June at the NSS than at SRS is just natural variability;

Line 446: Even greater than in Table 3? And why should the heat exchange be different at NSS and SRS if there is no snow? Because of the higher water content at NSS than at SRS? Then you need to argue which processes lead to the assumed result; however, 
it has to be consistent with your other findings;

Line 456: Instead of the grain size distribution it is rather the pore size distribution;

Line 457: Why and how? I would say the pore size distribution, the porosity and thus the bulk density are changed by digging, but not the grain size distribution;

Interactive

Line 462: Is it possible that the digging lead to preferential flow paths in the ground? However, you dug at both sites in the same way, right?

Lines 471-473: Does the annual rainfall of Delingha originate from 1960? Or was it only published then and is a long term mean, i.e. even older? It could have changed a lot since then! So you can't compare it with todays values of Yashatu;

Lines 480-481: Is half a page really necessary to come to this explanation?

Lines 483: Repetition of line $469 \rightarrow$ delete;

Line 483: 'the melt water equivalent to the SWE' instead of 'this result';

Line 483: '... could have increased ... by only $4.4 \%$...';

Line 493: Only in summer, when the evaporation is highest, the NSS was cooler than the SRS at $0.5 \mathrm{~m}$ depth (Fig. 8). However, then there was nearly no snow (Table 2). So I don't think that this effect contributed to the lower water content at SRS, otherwise the near surface temperatures ( $0.5 \mathrm{~m}$ depth) should have been higher at SRS than at NSS also in spring, winter and autumn which was not the case;

Line 497: But according to Tab. 2 there was nearly no snow in summer at your site;

Lines 498-499: But in Fig. 8 you have shown that the mean winter temperature is lower at the snow free SRS than at the snow covered NSS!

Printer-friendly version

Line 504: Also this sounds logical but should have resulted in lower near surface temperatures at NSS than at SRS, also in winter, spring and autumn. Or do you think this was the case, but you couldn't show it as no data could be gained above $0.5 \mathrm{~m}$ depth? 
Then you have to say it clearly;

Line 507: Which range do you mean - within a year? Then during the first year the VWC ranged between 0 and the highest class (40-?\%), in the second year between 0 and the second highest class (30\%-40\%). So what do the $50 \%$ decrease mean?

Line 508: '...as the snow removal duration increases' $\rightarrow$ does it mean 'as long as the snow removal will be continued'?

Line 515: The mean surface?

Lines 523-525: The topic of your paper are the effects of the seasonal snow cover on the hydrothermal conditions of the active layer; so here you should conclude that the snow removal at SRS lead to lower water contents which can be derived from the comparison with the NSS where the measurements took place at the same period of time facing the same (dry) meteorological conditions. So far (in point 3 of the conclusions) you argue only by the temporal sequence of 3 years;

Technical corrections:

Line 15: Replace '.' by ':';

Line 21: Instead of 'maybe results' use 'may result';

Line 36: Add space between 'Brown' and 'et', and 'Lemke' and 'et;'

Lines 47, 51: Avoid words like 'helpful' or 'help'; they indicate that you want something to be or not to be; however, as scientists there is no good or bad, you should just investigate nature without judging; otherwise you are subjective rather than objective;

Line 48: Why not simply 'snow surface'?

Printer-friendly version

Line 52: A physical property can't be good or bad;

Line 53: The $\mathrm{K}$ has to be big as it means Kelvin but not thousand;

Discussion paper

Line 56 : $\mathrm{K}$ instead of $\mathrm{k}$ in the unit $\mathrm{W} /(\mathrm{m} \cdot \mathrm{K} \cdot \mathrm{d})$;

C16 
Line 59: Add a space after the fullstop;

Line 60: '... with a thick ...'

Line 61: '... with a temperature difference of $20{ }^{\circ} \mathrm{C} \mathrm{...;}$

Line 64: '... that a snow cover ...;

Line 65: not 'will have' but 'has';

Line 65: '... a remarkable ....;

Line 67: I am not a native speaker, but the English in this sentence doesn't seem to be correct to me; check with a native speaker, please;

Line 69: '..., the seasonal snow cover ...;

Line 71: Add space between 10 and ${ }^{\circ} \mathrm{C}$;

Line 71: Add space after fullstop;

Line 74: Isn't it 'on the continent'? check with a native speaker, please;

Line 78: Add a point after 'al';

Line 82: Isn't it written CoupModel?

Line 83: I suggest 'strongly' rather than 'deeply'; check with native speaker;

Line 83: Add space after (2001);

Line 84: 'at' instead of 'over';

Line 84: '... the European Alps ...;

Line 84: '... that a $5-15 \mathrm{~cm}$ thick snow cover ...';

Line 88: Instead of 'melted snow' I suggest 'snow melt water'; besides the melt water originating from ground ice; 
Line 92: Put the acronym 'SNOW-17' after 'snow cover energy ... model';

Line 126 (Fig. 1d): Picture is slightly higher than that of Fig. 1c $\rightarrow$ adjust

Line 135: '... found (Figure 1d)';

Line 136: ‘ ... 20\% (Figure 1c). ';

Line 139: '... at the snow site ...';

Line 140: 'Mudstone occurs at a depth of $5.0 \mathrm{~m}$ for the NSS and $3.6 \mathrm{~m}$ for teh SRS (Figure 2)';

Line $144:$ '...-0.32 to $-0.30{ }^{\circ} \mathrm{C} . .$. ; then use âǍŽto' to indicate a range throughout the document;

Lines 148 and 150 (Fig. 2a and 2b): To make it clearer I recommend to omit the vertical lines between a property and its value, e.g. Altitude: $4040 \mathrm{~m}$;

Lines 148 and 150 (Fig. 2a and 2b): Regarding column 'Notes' $\rightarrow$ To save space in the figure itself you could give this information in the caption;

Line 151: Mark figure parts either with $a$ and $b$ and refer to them in the caption or with NSS and SRS but not both;

Line 153: September 2009?

Line 154: '... meteorological measurement equipment ...'

Line 154: November 2009?

Line 155: '... monitored using the sensors ...';

Line 157: '. . in May 2010 ...'

Line 162: '. . . in May 2010.';

Lines 162-163: 'A set of ... was installed ... of the two plots, NSS and SRS, respec- 
tively.'

Lines 165-168: '... were added at the NSS and the SRS, while an ultrasonic snow depth sensor and a sensor measuring the soil thermal flux at shallow depths were installed only at the NSS.';

Line 170 (Table 1): Use small letter at the beginning; also for soil temperature;

Line 170 (Table 1): Under the name 'types' you are mixing parameters (e.g. AT\&H) and measuring intsruments (e.g. barometer or rain gauge) $\rightarrow$ harmonize, please; use singular, e.g. 'Type of probe';

Line 170 (Table 1): 'Measuring range' instead of 'Ranges';

Line 170 (Table 1): 'Number of probes' instead of 'Numbers';

Line 170 (Table 1): In order to save space you could use small superscript numbers directly after the probe type;

Line 177: Add space: $6 \mathrm{~cm}$;

Line 178: '... are thus needed ...; then you can omit: 'because ... rapidly';

Line 182: '... to the automatic data logger CR3000.';

Line 183: instead of 'that ... when' use 'after';

Line 184: '... station, data were often not recorded during night at the SRS.';

Line 185: '... area differing between seasons.';

Line 191: Distance between the three pictures are slightly not the same as well as their height $\rightarrow$ adjust;

Line 193: 'soil temperature data acquisition' or 'soil temperature measurement';

Line 207: '... two parallel steel rods which are $300 \mathrm{~mm}$ long, $3.2 \mathrm{~mm}$ in diameter and separated by a distance of $32 \mathrm{~mm}$.'; 
Line 213: Not 'laid by drilling' but 'installed in a bore hole';

Line 214: 'installed' instead of 'laid';

Line 214: '... reaches its maximum thawing penetration.';

Line 216: 'or' instead of 'and';

Line 217: '... were thus due ...';

Line 219: '... extended from the ground surface to $3.6 \mathrm{~m}$ depth?';

Line 220: Not 'laid' but 'installed' (2 times in this line);

Line 224: Not 'by' but 'in'

Line 224: Instead of 'wouldn't' I would say 'shouldn't';

Line 226: '... water content in the thawed soil ...'?

Line 237: Why do you say 'surface snow cover' instead of simply 'snow cover'? As the snow cover is always deposited on the surface this word is dispensable;

Line 239 (Firg. 4): Curves are too thick (only in pdf?); add horizontal lines as visual orientation;

Lines 246, 248, 252: Use past tense because it occurred in 2014; check whole manuscript and use past tense for all events which were finished in the past;

Line 246: Check with editor if this date format is ok; However, the date format should be the same throughout the document which is not the case, e.g. line 245 (December 2013) differs from line 240 (2012.12);

Line 260: Add space after fullstop;

Line: 277: 'Figure 7' but not 'figure 7';

Line 280 (Fig. 7): Indicate at least one negative isotherm; 
Line 280 (Fig. 7): Mark figure parts either with a and b and refer to them in the caption or with NSS and SRS but not both;

Line 290: '... where the daily soil ...';

Line 298: 'could' instead of 'can';

Line 301: 'also' is dispensable;

Lines 329-330: '... removal, the mean annual ground temperature at $0.5 \mathrm{~m}$ and $2.0 \mathrm{~m}$ depth increased by $0.3^{\circ} \mathrm{C}$ and $0.2^{\circ} \mathrm{C}$, respectively.';

Line 334: No comma after 'namely';

Line 349: Don't make a new paragraph after only 1 sentence (minimum after 2);

Lines 350-351: 'At a depth of $0-50 \mathrm{~cm}$ soil moisture sites varies no more than $4 \%$ between the years 2012-2014 at each of the two sites.';

Line 351: 'soil moisture decreases with time, and' $\rightarrow$ not needed, delete;

Line 354: 'Between $80 \mathrm{~cm}$ and $120 \mathrm{~cm}$ depth, soil ...';

Line 372: '... at 0-5 cm depth is assumed to be the same as ...;

Line 390: Add space after Alps;

Line 391: Don't use first names for the citations in the text (only initials of first names in bibliography);

Line 401: A comma or 'and' is missing after 'snow removal';

Line 405: '... heat dissipation from the active layer to the atmosphere in winter (...).';

Line 407: Omit 'the';

Line 411: In addition to Fig. 6, also refer to Table 2;

Line 418 (Table 3, caption): The table is obviously not at the right place but should be 
positioned later;

Line 421: '... since the snow ...';

Line 423: '... of the active layer.';

Line 429: '... heat for water stored in a $1 \mathrm{~m} 3$ soil body at a volumetric water content (VWC) of $1 \%$ is $3350 \mathrm{~kJ} . .$. ;

Line 437: Add a space before 'According';

Line 464: 1 space before 'therefore' is engough;

Line 469: '...), resulting in a snow water equivalent (SWE) of ...';

Line 484: '... content between 0-2.5 $\mathrm{m}$ depth in the active layer ...;

Line 508: Add a space after the fullstop;

Line 524: '... SRS can be attributed to the removal of ...';

Line 628: Check that the authors' family names and the initials are always separated by commas in the bibliography (which is not the case e.g. in line 628 and 630)

Interactive comment on The Cryosphere Discuss., doi:10.5194/tc-2016-134, 2016. 